\title{
Misoprostol and termination of pregnancy: is there a need for ultrasound screening in a general population to assess the risk for adverse outcome in cases of uterine anomaly?
}

\author{
N. M. van der Veen · J. F. G. M. Brouns • \\ J. P. Doornbos $\cdot$ W. J. van Wijngaarden
}

Received: 5 March 2010/Accepted: 8 June 2010/Published online: 1 July 2010

(C) The Author(s) 2010. This article is published with open access at Springerlink.com

\begin{abstract}
Purpose and methods We reviewed the existing literature on medical termination of pregnancy in cases of congenital uterine malformation. Is medical termination of pregnancy safe in the presence of a uterine anomaly? Can termination of pregnancy still be performed when information concerning the presence of congenital uterine malformation is not available? Results The risk of adverse outcome, i.e. uterine rupture, was high in class 2 uterine anomalies, whereas the risks in classes 3-6 were negligible. However, the very low incidence of class 2 anomalies in pregnant women results in a calculated risk of uterine rupture in medical termination of pregnancy on the basis of this anomaly of 1 in 300,000 pregnancies. Ultrasound scanning is of limited diagnostic value to diagnose congenital uterine malformations.

Conclusions The implications of uterine anomalies are not an argument in the discussion whether to use misoprostol for termination of pregnancy in developing countries with scarce diagnostics tools.
\end{abstract}

Keywords Misoprostol · Uterine anomalies .

Diagnostic ultrasound scanning safety

\section{Introduction}

Of the 210 million pregnancies worldwide each year, $87 \%$ occurs in developing countries. Twenty-two million

N. M. van der Veen · J. F. G. M. Brouns $(\bowtie)$ · J. P. Doornbos Department of Obstetrics and Gynecology, Zaans Medical Centre, Julianaplein 58, 1502 DV Zaandam, The Netherlands e-mail: brouns.j@zaansmc.nl

W. J. van Wijngaarden

Department of Obstetrics and Gynecology, Bronovo Hospital, Bronovolaan 5, 2597 AX The Hague, The Netherlands terminations of pregnancy per year of a total of 42 million are legal within a local judicial system [1].

Medical abortion using prostaglandins became an alternative method for surgical abortion of first and second trimester pregnancies in the early 1970s [2]. This procedure appears to be an effective and safe method for abortion, although it might bear certain risks for women with a uterine anomaly.

The prevalence of congenital anomalies due to Müllerian duct malfusion has been estimated between $0.1 \%$ in an unselected population and $6 \%$ in women undergoing diagnostic imaging [3, 4].

In the Netherlands, these congenital anomalies are mostly detected before the termination of a pregnancy, as ultrasound investigation is routine in early pregnancy. However, detection is less likely in developing countries since diagnostic tools are scarce [5].

There is little knowledge about the risk of serious adverse outcome, i.e. uterine rupture in medical termination of pregnancy in the presence of a uterine anomaly [6, 7].

In this paper we present a case report and a review of the literature on the use of misoprostol for termination of pregnancy in women with uterine anomaly. Published risk percentages of uterine rupture in using misoprostol for medical abortion enabled us to calculate this risk in a population of pregnant women in developing countries requesting abortion.

\section{Background}

Uterine anomaly

A review on uterine anomalies by Nahum et al. [6] showed that congenital uterine anomalies affect $0.5 \%$ of women in 
Fig. 1 Classification of congenital uterine anomalies according to the American Fertility Society “AFS” (1988)

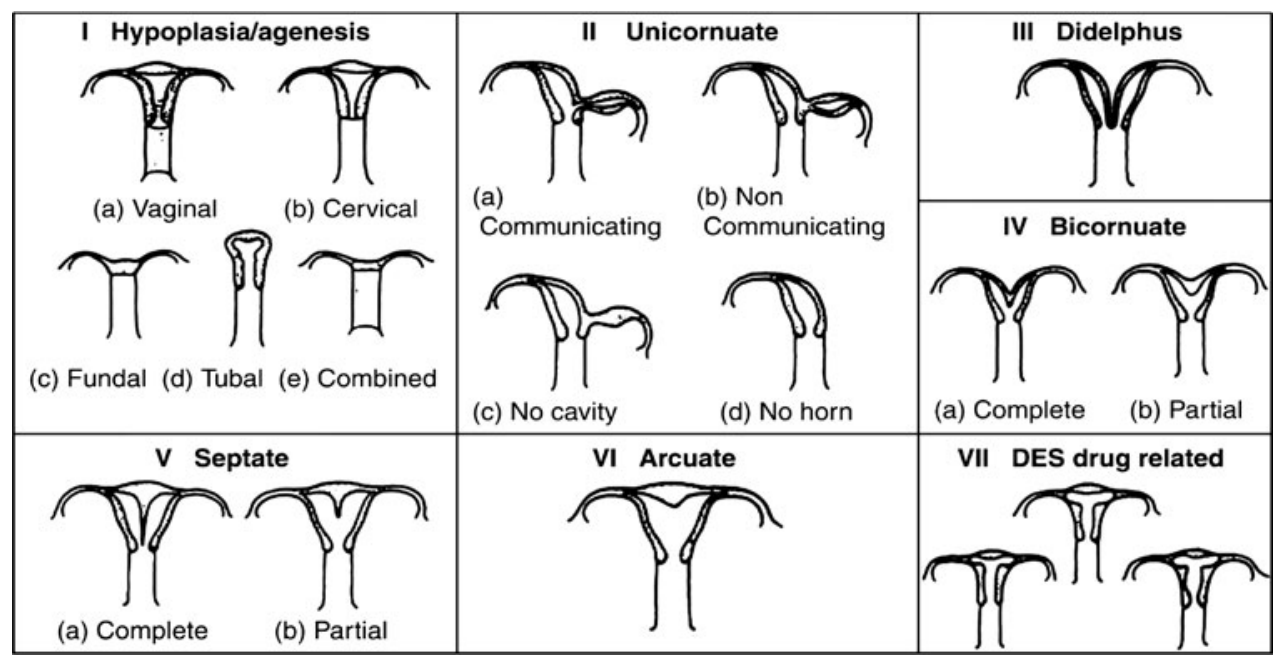

the general population and $0.2 \%$ in fertile women (47 studies were included in the review). The most common anomalies are the symmetric or duplication anomalies, including bicornuate uteri. Unicornuate uteri, resulting from unilateral failure of normal Müllerian system development, comprise $5 \%$ of all uterine anomalies. The vast majority of these have a contralateral rudimentary uterine horn of the non-communicating type, meaning that the endometrial cavity does not communicate with cervix, vagina, or contralateral hemi-uterus. This is often diagnosed at exploratory laparotomy after pregnancy related uterine rupture $[6,7]$.

In these rudimentary horns $(85 \%$ are non-communicating), approximately 1 in 76,000 pregnancies occur $[8,9]$. Fertilization in these cases takes place through transperitoneal migration of sperm from the patent contralateral fallopian tube [10].

\section{Prostaglandins}

In the 1990s, it became clear that misoprostol is an effective and cheap alternative to the traditionally used prostaglandins in obstetric practice. The way of action is similar to other prostaglandins although various natural and synthetic prostaglandins employ their action using different receptors and different biochemical pathways. The natural prostaglandins are rapidly metabolized in lung tissue. Synthetic prostaglandins, such as misoprostol, bypass the lung, thus prolonging their half-life.

Misoprostol has a strong uterotonic effect and is widely used in obstetrical and gynecological practice.

In comparison to the traditional prostaglandins such as dinoprostone and gemeprost used in obstetrics, misoprostol is cheap (one $0.2 \mathrm{mg}$ tablet costs US\$ $0.10-0.30$ ), easy to administer, and stable in light and hot climate conditions.
It can be kept on the shelf for long periods of time without losing any of its properties.

Misoprostol can be used safely for termination of pregnancy in first as well as in second trimester, in case of a viable and nonviable pregnancy [2,11-13]. It is a safe and cost effective alternative to surgical abortion, in resource poor countries [14].

\section{Risk assessment}

Women with major uterine anomalies have an increased risk for uterine rupture during pregnancy and delivery [15]. This may be due to a developmental inferior musculature of the uterine wall. Myometrial thickness decreases with gestational age and, in addition, the uterine wall becomes thinner as a result of uterine contractions $[8,9,15]$.

These anatomical phenomena will probably be less important in cases of symmetric anomalies such as didelphic, bicornuate, and septate uteri. However, for pregnancies in (non-)communicating rudimentary uterine horns, vaginal delivery is impossible and potentially dangerous [15]. Induction of myometrial contractions is potentially hazardous in such cases as uterine rupture may ensue.

If we consider all different subtypes according to the classification of congenital uterine anomalies of the American Fertility Society "AFS" (1988, Fig. 1) it is especially class II that poses an increased risk. Patients with a class I anomaly have no reproductive potential, class III and IV hardly have obstetrical complications and class VI is considered a normal variant. Although the septate uterus (Class V) is correlated with reproductive complications, there is no risk of uterine rupture since the musculature is normally developed.

The prognosis of pregnancies in a rudimentary uterine horn is poor with regard to both maternal and fetal outcome (neonatal survival rate of $6 \%$ and maternal mortality rate of 


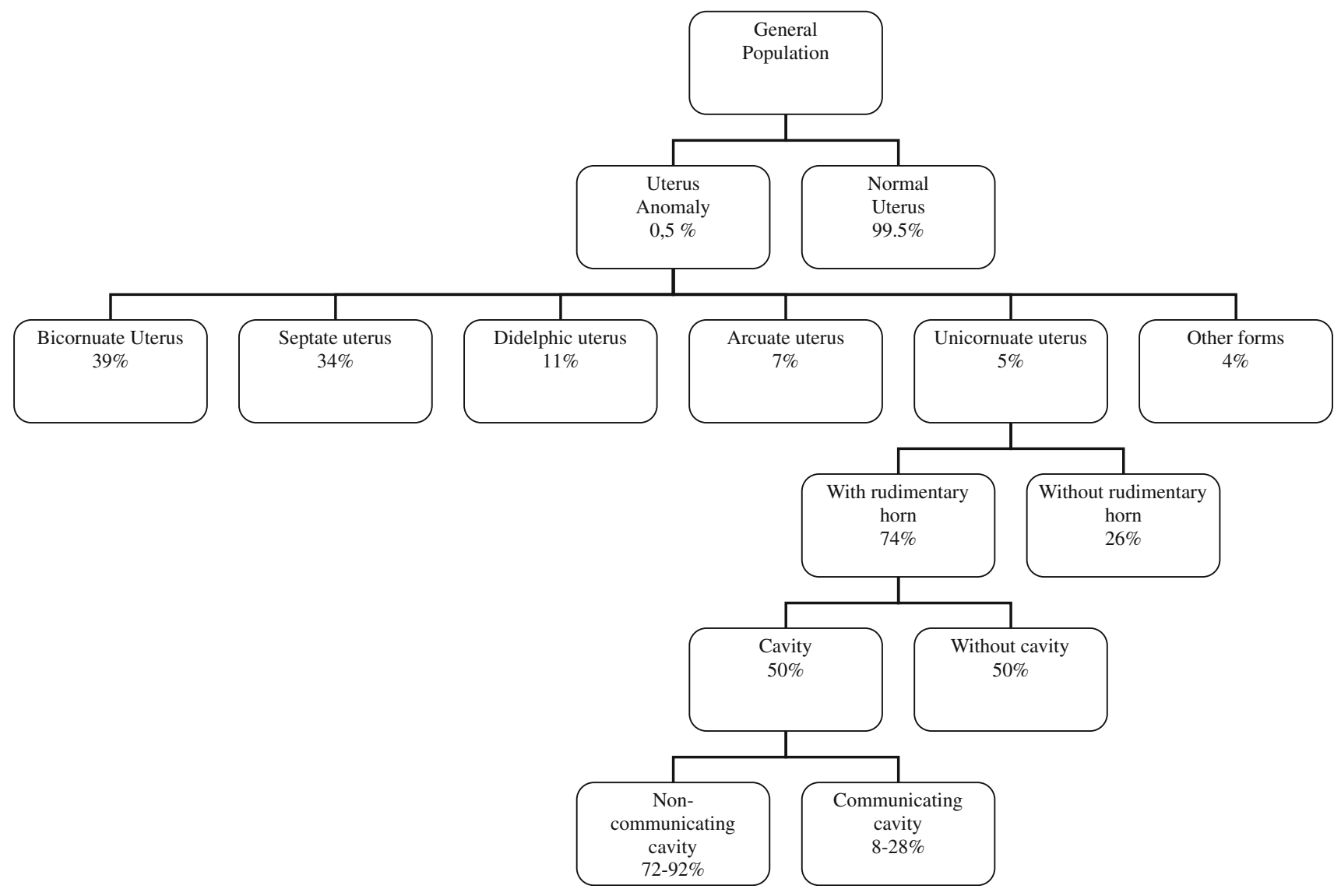

Fig. 2 Distribution of uterine anomalies [8]

5.7 and $1.9 \%$ for non-communicating and communicating horns, respectively) [9]. Rupture of the rudimentary horn accounts for $82 \%$ of these maternal mortalities.

Nahum et al. mentioned $47 \%$ of reported pregnancies in non-communicating horns to be ruptured, as did $52 \%$ of the communicating horns resulting in an overall risk of about $50 \%$. Of these ruptures, $67 \%$ occurred during the second trimester and $13 \%$ in the first trimester [9]. Induction of uterine contractions with misoprostol is likely to cause uterine rupture, especially in the second trimester. The prevalence of uterine anomalies is 1 in 200 in the general female population and 1 in 590 in the fertile female population [6]. As the estimated risk of the class II uterine anomaly is $5 \%$ (Figs. 2, 3), the prevalence of class II anomaly is $1 / 11880$ fertile women (Figs. 2, 3). Pregnancy in a rudimentary horn occurs in $1 / 76,000$ [16]. Little is known about racial differences in the prevalence of congenital uterine malformations.

With a sensitivity of ultrasound scanning to detect a case of rudimentary horn of $26 \%$ [7], 292,307 scans have to be made to prevent one possible case of uterine rupture (assuming the risk of rupture is $100 \%$ in case of a rudimentary horn).

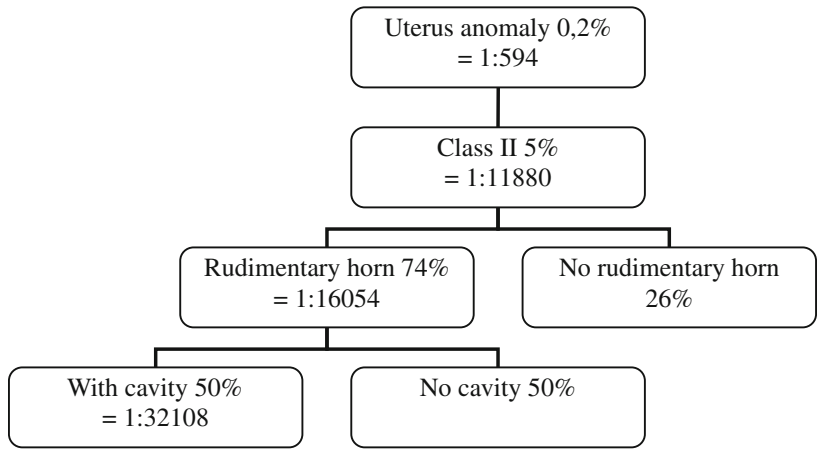

Fig. 3 Prevalence of uterine anomalies in fertile women

\section{Case}

A 32-year-old primigravida presented herself at our outpatient department requesting a termination of pregnancy. Ultrasound scan revealed a vital intrauterine pregnancy of 8 weeks and a uterine anomaly in the form of a class II or a class III malformation (unicornuate uterus with a rudimentary horn or a uterus didelphys). Generally in our clinic all women requesting a termination of pregnancy are counseled for a surgical as well as a medical procedure 
while taking all contraindications into consideration. Although our patient preferred a medical procedure, the attending physician doubted the safety of medical termination with misoprostol, deciding upon the surgical procedure instead.

Literature search

A systematic search was performed in PubMed and Cochrane, using the search terms: "uterine anomaly," "uterine abnormalities," “misoprostol," “Müllerian anomaly," "rudimentary uterine horn," "unicornuate uterus," and "bicornuate uterus".

Only three case reports and one accompanying report have been published which matched our search.

In two of these case reports, termination of pregnancy with misoprostol resulted in uterine rupture of the noncommunicating rudimentary horn $[17,18]$. Chao et al. [19] report the case of an incomplete medical abortion in a twin pregnancy in a bicornuate uterus. Nahum et al. [15] suggest a development of a set of practical guidelines.

\section{Discussion}

Since the introduction of misoprostol, there has been concern about its safety. The manufacturer has sent several warning letters against the unlicensed use of misoprostol during pregnancy [20].

Risks concerning the use of misoprostol are its teratogenic properties, hyperstimulation of the uterus, pregnancy loss and uterine rupture. Most of these safety issues have been addressed. Regimens used in obstetric and gynecological practice that are in accordance with professional guidelines are considered effective and safe [13, 21].

These guidelines, based on best available evidence, presume that the medical workup before a termination of pregnancy is carried out, is up to standard. Physical examination and access to ultrasound diagnostics are essential in this. Ultrasound scanning can give an accurate estimation of gestational age, reducing the incidence and severity of complications [22], but can also reveal the presence of uterine anomalies. Unfortunately, sensitivity of ultrasound scanning is only $26 \%$ in cases of corneal pregnancy [7]. Generally the identification of uterine anomalies is difficult and will need well-trained personnel. Criteria for recognition have been listed by Mavrelos et al. [23]. During an established pregnancy, ascertaining the exact type of mullerian anomaly is extremely challenging, with a definite diagnosis of the subclass being possible only outside pregnancy.

Most pregnant women in developing countries do not have access to ultrasound investigations or even physical examination by skilled professionals [1,24]. Even so, a class II anomaly can be easily missed on routine examination in the absence of an associated cervical or vaginal abnormality [6]. Particularly for this group of women, it is important to know whether the administration of misoprostol is safe without any knowledge on the presence of a uterine anomaly.

\section{Conclusion}

The precise risks of uterine rupture with misoprostol for termination of pregnancy in the presence of uterine anomalies are not known. Only a few case reports are available. There is still a lack of evidence of safety for the use of misoprostol in settings where diagnostic and therapeutic tools are scarce. The relatively low frequency of these uterine anomalies indicates that on a large scale, the drug is safe, but the exact rate of complications in women affected by Mullerian anomalies remains unknown and is possibly higher than in the general population. Calculations on the prevalence of uterine malformations and the incidence of possibly related serious complications in cases of termination of pregnancy support our opinion that diagnostic ultrasound scanning prior to medical termination of pregnancy to rule out a potentially dangerous uterine anomaly is not mandatory, although large scale studies would be needed to provide a definitive answer toward the need of ultrasound scanning.

Caring for pregnant women with congenital uterine malformations can be particularly challenging, with increased risks of complications arising from surgical and medical ToPs alike. These women might benefit from individualized protocols (e.g. lower doses, use of mifepristone prior to misoprostol).

\section{Conflict of interest statement None.}

Open Access This article is distributed under the terms of the Creative Commons Attribution Noncommercial License which permits any noncommercial use, distribution, and reproduction in any medium, provided the original author(s) and source are credited.

\section{References}

1. World Health Organization (2007) Unsafe abortion: global and regional estimates of the incidence of unsafe abortion and associated mortality in 2003. WHO, Geneva

2. Kulier R, Gulmezoglu AM, Hofmeyr GJ, Cheng LN, Campana A (2004) Medical methods for first trimester abortion. Cochrane Database Syst Rev (2):CD002855

3. Jermy K, Oyelese O, Bourne T (1999) Uterine anomalies and failed surgical termination of pregnancy: the role of routine preoperative transvaginal sonography. Ultrasound Obstet Gynecol 14(6):431-433 
4. Oláh KS (2002) Uterine torsion and ischaemia of one horn of a bicornute uterus: a rare cause of failed second trimester termination of pregnancy. BJOG 109(5):585-586

5. Harris RD, Marks WM (2009) Compact ultrasound for improving maternal and perinatal care in low-resource settings: review of the potential benefits, implementation challenges, and public health issues. J Ultrasound Med 28(8):1067-1076

6. Nahum GG (1998) Uterine anomalies. How common are they, and what is their distribution among subtypes? J Reprod Med 43(10):877-887

7. Jayasinghe Y, Rane A, Stalewski H, Grover S (2005) The presentation and early diagnosis of the rudimentary uterine horn. Obstet Gynecol 105(6):1456-1467

8. Nahum GG (1997) Rudimentary uterine horn pregnancy. A case report on surviving twins delivered eight days apart. J Reprod Med 42(8):525-532

9. Nahum GG (2002) Rudimentary uterine horn pregnancy. The 20th-century worldwide experience of 588 cases. J Reprod Med 47(2):151-163

10. Nahum GG, Stanislaw H, McMahon C (2004) Preventing ectopic pregnancies: how often does transperitoneal transmigration of sperm occur in effecting human pregnancy? BJOG 111(7):706714

11. Gomez Ponce de LR, Wing DA (2009) Misoprostol for termination of pregnancy with intrauterine fetal demise in the second and third trimester of pregnancy - a systematic review. Contraception 79(4):259-271

12. Neilson JP, Hickey M, Vazquez J (2006) Medical treatment for early fetal death (less than 24 weeks). Cochrane Database Syst Rev 3:CD002253
13. The Royal College of Obstetricians, Gynaecologists (2004) The care of women requesting induced abortion. RCOG Press, London

14. Bergstrom S, Aronsson A (2008) Misoprostol in resource poor countries. BMJ 336(7652):1032

15. Nahum GG (2005) Uterine anomalies, induction of labor, and uterine rupture. Obstet Gynecol 106(5 Pt 2):1150-1152

16. Nahum GG (1999) Rudimentary uterine horn pregnancy. J Reprod Med 44(3):314-315

17. Bradshaw H, Stewart P (2004) Failed medical termination of pregnancy associated with implantation in a non-communicating uterine horn. J Fam Plann Reprod Health Care 30(3):178

18. Samuels TA, Awonuga A (2005) Second-trimester rudimentary uterine horn pregnancy: rupture after labor induction with misoprostol. Obstet Gynecol 106(5 Pt 2):1160-1162

19. Chao A, Chao AS, Wang ST, Wang TH (2006) Mifepristoneinduced abortion in one horn and a growing fetus in another horn in a patient with a twin pregnancy in a bicornuate uterus. Fertil Steril 86(6):1764-1765

20. Friedman MA (2001) Manufacturer's warning regarding unapproved uses of misoprostol. N Engl J Med 4(344):1-61

21. The American College of Obstetricians and Gynecologists (2001) Medical management of abortion. ACOG Press, Washington

22. Burnhill MS, Armstead JW (1978) Reducing the morbidity of vacuum aspiration abortion. Int J Gynaecol Obstet 16(3):204-209

23. Mavrelos D, Sawyer E, Helmy S, Holland TK, Ben-Nagi J, Jurkovic D (2007) Ultrasound diagnosis of ectopic pregnancy in the non-communicating horn of a unicornuate uterus (cornual pregnancy). Ultrasound Obstet Gynecol 30(5):765-770

24. World Health Organization (2005) World Health Report 2005. Make every mother and child count. WHO, France 